\section{Telestroke en Chile: resultados de 1 año de experiencia de la Unidad de TeleACV del Servicio de Salud Metropolitano Sur en 7 hospitales ejecutores}

\author{
ELOY MANSILLA ${ }^{1,2,3}$, ENRICO MAZZON ${ }^{1,3}$, DANIEL CÁRCAMO ${ }^{1,3}$, \\ FELIPE JURADO ${ }^{1,2}$, LORENA LARA ${ }^{1}$, MIRYAARÉVALO $^{1, a}$, DIEGO ROJAS $^{1, \mathrm{~b}}$, \\ GLORIA STEPHENS ${ }^{1, \mathrm{a}}$, ARNOLD HOPPE ${ }^{3}$, ALEJANDRO BRUNSER $^{3}$
}

\section{Telestroke in Chile: 1 year experience at 7 hospitals}

Background: Acute ischemic stroke (AIS) is one of the leading causes of death in Chile. Intravenous thrombolysis (IVT) is an effective treatment. Geographical barriers and lack of specialists limit its application. Telemedicine can overcome some of these pitfalls. Aim: To describe the implementation and results of AIS treatment by telemedicine at the TeleStroke Unit (TeleACV) of the Southern Metropolitan Health Service, connected with seven hospitals in Chile. Material and Methods: Descriptive analysis of a prospective tele-thrombolysis data-base that covers from 2016 to 2018, with an emphasis in the last year. Results: During the analyzed period, seven remote telemedicine centers were activated as far as 830 kilometers on a continental level from the reference center and up to 3,700 kilometers on an island level. There were 1,024 telemedicine consultations, 144 (14\%) of them resulted in an IVT treatment. During 2018, 597 tele-consultations were made, thrombolysis was done in 115 (19\%) patients aged 66+-13 years; $54(46.6 \%)$ being female. The median admission National Institute of Health Stroke Scale was 8 (interquartile range (IQR) 5-14). The median door-to-needle time was 56.5 (IQR 44.8-73.3) minutes; 60\% of patients were treated within 60 minutes. Eight patients (7\%) were referred for a subsequent mechanical thrombectomy to a center of greater complexity. Symptomatic intra-cranial hemorrhages occurred in four treated patients (4\%). One patient had a systemic bleeding. Conclusions: The Telestroke Unit achieved a high rate of IVT and good door-to-needle times. This may help to overcome some of the geographic barriers and the specialist availability gap in our country.

(Rev Med Chile 2019; 147: 1107-1113)

Key words: Fibrinolysis; Thrombolytic Therapy; Stroke; Remote Consultation; Telemedicine; Chile.



E 1 accidente cerebrovascular isquémico (ACVi) es la principal causa de muerte y discapacidad en Chile ${ }^{1}$ y la segunda causa de muerte en el mundo ${ }^{2}$. La trombolisis intravenosa (TIV) hasta las $4,5 \mathrm{~h}$ de evolución es la principal terapia de reperfusión aprobada para el $\mathrm{ACVi}^{3,4}$, con excelentes resultados, obteniendo un paciente adicional independiente por cada 7-8 enfermos $\operatorname{tratados}^{3,5}$. La falta de neurólogos y las limitaciones geográficas impiden su aplicación masiva en muchos lugares del mundo. El uso de la telemedicina para superar estas barreras fue propuesto 
en 1999 por Levine y Gorman ${ }^{6}$ y se inició como experiencia piloto en Baviera en el año 2005 ${ }^{7}$. Su uso se ha expandido con diversas modalidades en varios países ${ }^{8-13}$, reportando resultados similares al tratamiento asistido por un neurólogo presencial e incluso mejorando los tiempos de acción y aumentando las tasas de trombolisis ${ }^{14}$, consolidándose como una estrategia costo-efecti$\mathrm{va}^{15}$ con parámetros de calidad bien definidos ${ }^{16} \mathrm{y}$ siendo incorporada en guías clínicas de manejo del ACVi como las de la American Stroke Association ${ }^{17}$. Sin embargo, no todas las experiencias han sido exitosas y ciertas condicionantes locales pueden determinar su fracaso ${ }^{18,19}$.

Nuestro objetivo es describir la aplicación de un programa de teleasistencia de ACVi y los resultados de los pacientes con ACVi trombolizados por la Unidad de TeleACV (UTA) del Servicio de Salud Metropolitano Sur (SSMS) con varios hospitales del país.

\section{Pacientes y Método}

A mediados de 2014, se planteó al SSMS el desarrollo de un sistema de telemedicina para el ACVi. Para esto se visitaron centros hospitalarios que tuvieran interés en instalar un sistema de diagnóstico y tratamiento del ACVi agudo por telemedicina. Los centros debían tener una disponibilidad para realizar estudios de imagen con tomografía computada (TC) las 24 h del día, los 7 días de la semana (24/7) y carecer de neurólogos en sus unidades de urgencia. El sistema se inició con una experiencia piloto con el Hospital El Pino del área sur de Santiago. La UTA se constituyó como tal en octubre de 2017 al aumentar el número de hospitales bajo el sistema y al obtener el apoyo del Ministerio de Salud con cargos de neurólogos $28 \mathrm{~h}$ y equipo multidisciplinario. En los meses siguientes se incorporaron sucesivamente 7 hospitales al sistema de TeleACV. En estos centros se realizó un meticuloso levantamiento de las condiciones físicas, profesionales y de los liderazgos. Se procedió a realizar modificaciones físicas en los servicios de urgencia, tales como instalación de puntos de red y adaptación del box para telemedicina. Además, se desarrollaron protocolos y flujogramas de atención médica y de enfermería, se realizaron reuniones de docencia con el personal clínico y administrativo incluyendo la enseñanza de las escalas de triage y de gravedad del ACVi, capacitación sobre los beneficios y los riesgos de la TIV, así como los criterios de inclusión y exclusión para este tratamiento.

De acuerdo con el flujograma, cuando un paciente concurre al hospital ejecutor con síntomas sugerentes de un ACVi se aplica un tamizaje basado en la Escala de Cincinatti ${ }^{20}$; de existir asimetría facial, pérdida en la fuerza de un brazo/ pierna o alteración de lenguaje, se activa el "código ACV”. Bajo este código, el paciente es llevado inmediatamente a un box, se toman exámenes de sangre, el médico de urgencia confirma la sospecha diagnóstica de enfermedad cerebrovascular y procede a catalogar la gravedad del paciente con el puntaje otorgado por la escala del National Institute of Health Stroke Scale o NIHSS ${ }^{21}$. Se realiza una tomografía computada de cerebro sin contraste a la brevedad y se comunica vía telefónica a la UTA, donde existe un neurólogo de turno solo para consultas de TeleACV en horario 24/7. Posteriormente, el paciente es llevado a un box especialmente equipado con un dispositivo móvil de telemedicina (DMT), que consiste en un equipo de videoconferencia con vista panorámica del paciente, campo visual mínimo de $120^{\circ}$ con posición reajustable y calidad de servicio (con priorización de tráfico y ancho de banda) en punto de red del box de telemedicina. El neurólogo puede ver e interrogar al paciente, dirigir el examen realizado por el médico de urgencia y acceder al repositorio de radiología para observar las neuroimágenes y obtener la escala de ASPECTS ${ }^{22}$.

Con toda la información clínica, el neurólogo procede a tomar la decisión de indicar o no el tratamiento trombolítico. En caso de aplicar tratamiento fibrinolítico, se utiliza alteplase a una dosis de $0,9 \mathrm{mg} / \mathrm{kg}$ hasta un máximo de 90 $\mathrm{mg}$, pasando $10 \%$ de la dosis en bolo en $1 \mathrm{~min}$ y el resto de la dosis en $1 \mathrm{~h}$. Durante la hora de infusión del trombolítico, el neurólogo participa a distancia en el manejo del paciente junto al equipo de urgencia.

Los cuidados postrombolisis se realizan de acuerdo a los protocolos locales utilizando como referencia el estudio NINDS ${ }^{3}$, siendo el paciente trasladado a una unidad de cuidados especiales del mismo hospital y, solo en caso de requerir otra intervención especializada, es trasladado a un centro de referencia. Además, el paciente es reevaluado entre las 3 y $24 \mathrm{~h}$ postrombolisis por 
un neurólogo de la UTA o por un neurólogo presencial en horario hábil del hospital ejecutor en caso de contar con ese recurso.

En una base de datos anonimizada se registran los datos más relevantes para un adecuado control de calidad del sistema de telemedicina: edad y sexo del paciente, hospital ejecutor, gravedad del ACVi por la escala de NIHSS, tiempo transcurrido desde inicio de síntomas al arribo del paciente al servicio de urgencia, tiempo transcurrido desde el ingreso del paciente a la TC cerebral, hora de activación del código ACV, eventual aplicación de la TIV y tiempo puerta-aguja (tiempo transcurrido desde el ingreso del paciente al servicio de urgencia hasta recibir el bolo de trombolítico). Se registran las eventuales complicaciones de tratamiento, como hemorragia cerebral sintomática, definida como la presencia de una hemorragia cerebral en una TC de cerebro asociada a un deterioro clínico de $\geq 4$ puntos en la escala de NIHSS durante las primeras $24 \mathrm{~h}$ de evolución del enfermo sin otra causa que explique el deterioro neurológico. También son registradas las hemorragias extracraneales mayores, de acuerdo a los criterios del estudio GUSTO ${ }^{23}$.

La realización de este estudio y sus respectivos análisis fueron aprobados por el Comité Ético Científico del SSMS.

\section{Análisis estadístico}

Análisis descriptivo de una base de datos colectada prospectivamente durante el período 2016 a 2018. Además, se analiza separadamente el año calendario 2018. Se describe la cantidad de llamadas de telemedicina efectuadas durante el tiempo de estudio y el porcentaje de pacientes con ACVi que fueron trombolizados. Se describen los tiempos transcurridos desde el ingreso del paciente al servicio de urgencia a la TC cerebral, la activación del código ACV y puerta-aguja (ingreso a servicio de urgencia hasta comenzar a pasar el fármaco trombolítico). Se calcula el porcentaje de complicaciones hemorrágicas intracraneanas y extracraneanas en los pacientes tratados con TIV.

\section{Resultados}

El primer hospital incluido en el programa de TeleACV fue el Hospital El Pino, en febrero de 2016 y el último el Hospital de Hanga Roa (Rapa Nui) en diciembre de 2018. Durante 2018 se in- corporaron, además, los hospitales de Copiapó, Vallenar, Talagante, Quillota y San Antonio. La mayoría de los hospitales aquí presentados son considerados como de mediana complejidad desde el punto de vista de su capacidad resolutiva, sin embargo, la patología que se recibe en urgencias no hace distinción de gravedad. El Hospital de Copiapó está definido como un hospital de alta complejidad.

Durante el período de análisis se realizaron 1.024 atenciones de telemedicina, las que fueron aumentando progresivamente con el transcurso del tiempo (Figura 1a y 1b). Un total de 144 (14\%) atenciones resultaron en un tratamiento con TIV.

El análisis del año 2018, correspondiente a los 12 meses más activos del sistema de telemedicina, con casi $60 \%$ del total de las atenciones y con los 7 hospitales ejecutores activados, muestra que $96 \%$ (576 de 597) de las atenciones fueron videoconferencias sincrónicas en presencia del paciente, el médico de urgencias y personal de enfermería. La minoría de las atenciones se realizó solo entre profesionales, es decir, llamadas telefónicas simples o videoconferencias sin el enfermo presente (Tabla 1). Todas las TIV se realizaron de manera sincrónica. Las características de los pacientes trombolizados en el año 2018 puede ser observada en la Tabla 2. La media de edad de los pacientes consultantes fue de 65,6 años (DS 13,2), $54(46,6 \%)$ pacientes fueron de sexo femenino y la mediana de NIHSS de ingreso fue de 8 (RIQ 5-14). El tiempo puerta a TC cerebral fue de 14 min (RIQ 5-26), el tiempo puerta-videoconferencia de $26 \mathrm{~min}$ (RIQ 15-39) y el tiempo puerta-aguja de 56 (RIQ 45-73) minutos, con 56\% de los pacientes tratados antes de los $60 \mathrm{~min}$. Ocho pacientes $(7 \%)$ consultantes en centros cercanos a Santiago fueron derivados para una posterior trombectomía mecánica a un centro de mayor complejidad. Solo en $4(3,8 \%)$ pacientes trombolizados el año 2018 se presentó una hemorragia intracraneana sintomática. Un paciente cursó una hemorragia digestiva.

\section{Discusión}

Este estudio muestra que un programa de telemedicina en ACVi como el descrito puede implementarse en nuestro país y tiene el potencial de estrechar las brechas de acceso, tanto geográ- 




Figura 1a.

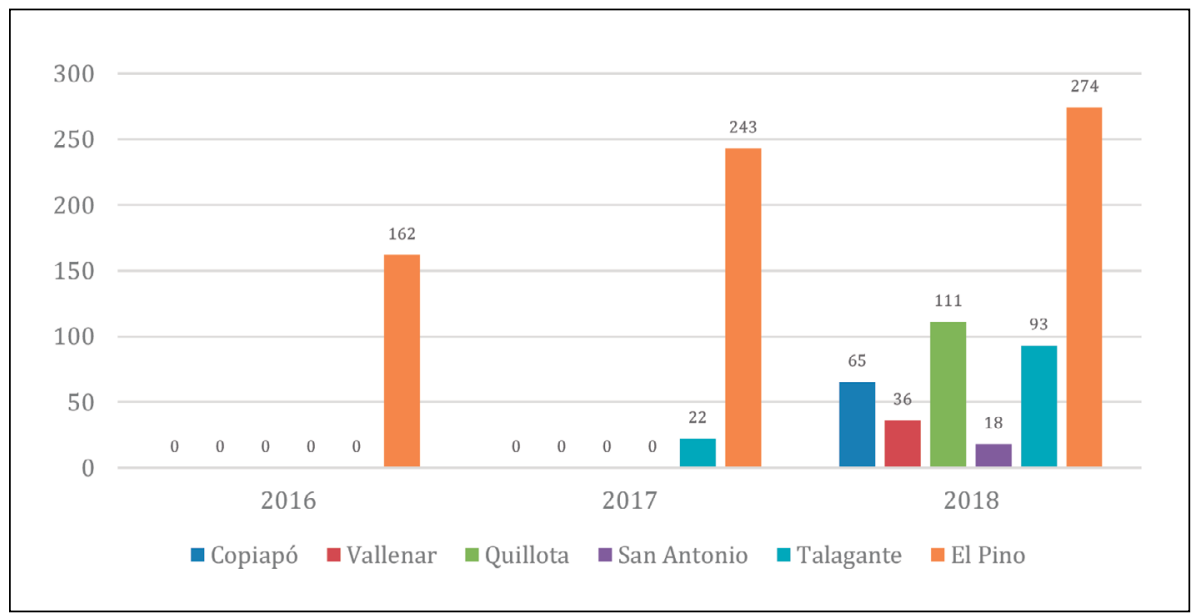

Figura 1b.

Tipo de atenciones realizadas durante 2018, por hospital ejecutor

\begin{tabular}{|c|c|c|c|c|c|c|c|}
\hline & $\begin{array}{c}\text { Total } \\
597 \\
(100 \%)\end{array}$ & $\begin{array}{c}\text { Copiapó } \\
65 \\
(10,9 \%)\end{array}$ & $\begin{array}{c}\text { Vallenar } \\
36 \\
(6 \%)\end{array}$ & $\begin{array}{c}\text { Quillota } \\
111 \\
(18,6 \%)\end{array}$ & $\begin{array}{c}\text { San Antonio } \\
18(3 \%)\end{array}$ & $\begin{array}{c}\text { Talagante } \\
93 \\
(15,6 \%)\end{array}$ & $\begin{array}{c}\text { El Pino } \\
274 \\
(45,9 \%)\end{array}$ \\
\hline Sincrónicas sin el paciente & 21 & 5 & 4 & 4 & 0 & 3 & 5 \\
\hline Video conferencia & 4 & $\mathrm{n} / \mathrm{r}^{*}$ & $\mathrm{n} / \mathrm{r}^{*}$ & $\mathrm{n} / \mathrm{r}^{*}$ & $\mathrm{n} / \mathrm{r}^{*}$ & $\mathrm{n} / \mathrm{r}^{*}$ & 4 \\
\hline Telefónicas & 17 & 5 & 4 & 4 & 0 & 3 & 1 \\
\hline $\begin{array}{l}\text { Sincrónicas video conferencias } \\
\text { con el paciente }\end{array}$ & 576 & 60 & 32 & 107 & 18 & 90 & 269 \\
\hline
\end{tabular}

Las atenciones corresponden a 3 tipos de prestaciones: Código ACV, controles de código ACV previamente atendidos e interconsultas de pacientes no código ACV. Todos los códigos ACV se atienden de forma sincrónica con el paciente vía videoconferencia. ${ }^{*} \mathrm{n} / \mathrm{r}$ : no registra. 
Tabla 2. Características e indicadores de calidad de los pacientes trombolizados en 2018 por hospital ejecutor

\begin{tabular}{|c|c|c|c|c|c|c|c|}
\hline & $\begin{array}{l}\text { Total } \\
\text { n: } 115\end{array}$ & $\begin{array}{l}\text { Copiapó } \\
\text { n: } 16\end{array}$ & $\begin{array}{l}\text { Vallenar } \\
\text { n: } 5\end{array}$ & $\begin{array}{l}\text { Quillota } \\
\text { n: } 32\end{array}$ & $\begin{array}{l}\text { San Antonio } \\
\text { n: } 6\end{array}$ & $\begin{array}{c}\text { Talagante } \\
\text { n: } 27\end{array}$ & $\begin{array}{l}\text { El Pino } \\
\text { n: } 29\end{array}$ \\
\hline Edad $\left(D^{\dagger}\right)$ & $\begin{array}{c}65,6 \\
(13,2)\end{array}$ & $\begin{array}{c}70,4 \\
(13,2)\end{array}$ & $\begin{array}{c}61,4 \\
(11,2)\end{array}$ & $\begin{array}{c}65,1 \\
(12,8)\end{array}$ & $\begin{array}{c}61 \\
(14,2)\end{array}$ & $\begin{array}{c}66,1 \\
(12,9)\end{array}$ & $\begin{array}{c}64,5 \\
(14,4)\end{array}$ \\
\hline Sexo femenino (\%) & $\begin{array}{c}54 \\
(46,9)\end{array}$ & $\begin{array}{c}8 \\
(50,0)\end{array}$ & $\begin{array}{c}2 \\
(40,0)\end{array}$ & $\begin{array}{c}11 \\
(34,4)\end{array}$ & $\begin{array}{c}3 \\
(50,0)\end{array}$ & $\begin{array}{c}13 \\
(48,2)\end{array}$ & $17(58,6)$ \\
\hline $\begin{array}{l}\text { NIHSS ingreso* mediana y rango } \\
\text { intercuartil }\end{array}$ & $\begin{array}{c}8 \\
(5-14)\end{array}$ & $\begin{array}{l}10,5 \\
(6-14)\end{array}$ & $\begin{array}{c}4 \\
(4-10)\end{array}$ & $\begin{array}{c}7 \\
(4-14)\end{array}$ & $\begin{array}{c}12,5 \\
(5-15)\end{array}$ & $\begin{array}{c}8 \\
(6-15)\end{array}$ & $\begin{array}{c}9 \\
(6-12)\end{array}$ \\
\hline $\begin{array}{l}\text { NIHSS } 60 \text { min* mediana y rango } \\
\text { intercuartil }\end{array}$ & $\begin{array}{c}6 \\
(3-11)\end{array}$ & $\begin{array}{c}8,5 \\
(4,5-12)\end{array}$ & $\begin{array}{c}4 \\
(4-5)\end{array}$ & $\begin{array}{c}6 \\
(2-8,5)\end{array}$ & $\begin{array}{c}6 \\
(2-13)\end{array}$ & $\begin{array}{c}6 \\
(3-15)\end{array}$ & $\begin{array}{c}5 \\
(2-8)\end{array}$ \\
\hline $\begin{array}{l}\text { Tiempo puerta a aguja, min* } \\
\text { (DS) }\end{array}$ & $\begin{array}{c}56,5 \\
(45-73)\end{array}$ & $\begin{array}{c}58 \\
(47-85)\end{array}$ & $\begin{array}{c}69 \\
(52-85)\end{array}$ & $\begin{array}{c}45,5 \\
(36,5-56,5)\end{array}$ & $\begin{array}{c}61,5 \\
(54-74)\end{array}$ & $\begin{array}{c}63 \\
(48-78)\end{array}$ & $\begin{array}{c}63 \\
(48-73)\end{array}$ \\
\hline $\begin{array}{l}\text { Tiempo síntomas a aguja min* } \\
\text { (DS) }\end{array}$ & $\begin{array}{c}159 \\
(110-207)\end{array}$ & $\begin{array}{c}140 \\
(101-185)\end{array}$ & $\begin{array}{c}145 \\
(78-168)\end{array}$ & $\begin{array}{c}130 \\
(130-214)\end{array}$ & $\begin{array}{c}218 \\
(141-267)\end{array}$ & $\begin{array}{c}157,5 \\
(119-205)\end{array}$ & $\begin{array}{c}158 \\
(90-194)\end{array}$ \\
\hline $\begin{array}{l}\text { Tiempo puerta a tomografía } \\
\text { computada, min* (DS) }\end{array}$ & $\begin{array}{c}14 \\
(5-26)\end{array}$ & $\begin{array}{c}14 \\
(7-25)\end{array}$ & $\begin{array}{c}32 \\
(12-54)\end{array}$ & $\begin{array}{c}5 \\
(2,5-14)\end{array}$ & $\begin{array}{c}9 \\
(8-13)\end{array}$ & $\begin{array}{c}12 \\
(3-26)\end{array}$ & $\begin{array}{c}27 \\
(20-34)\end{array}$ \\
\hline 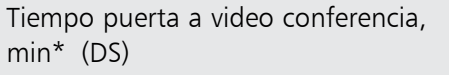 & $\begin{array}{c}26 \\
(15-39)\end{array}$ & $\begin{array}{c}30 \\
(10-57)\end{array}$ & $\begin{array}{c}18 \\
(16-44)\end{array}$ & $\begin{array}{c}20 \\
(12-28)\end{array}$ & $\begin{array}{c}38 \\
(26-39)\end{array}$ & $\begin{array}{c}25,5 \\
(15-40)\end{array}$ & $\begin{array}{c}31 \\
(19-39)\end{array}$ \\
\hline Trombectomía (\%) & $8(7,0)$ & $0(0,0)$ & $0(0,0)$ & $1(3,1)$ & $0(0,0)$ & $4(15,0)$ & $3(10,3)$ \\
\hline Hemorragia cerebral sintomática (\%) & $4(3,8)$ & $0(0,0)$ & $0(0,0)$ & $2(6,3)$ & $0(0,0)$ & $1(3,7)$ & $1(3,5)$ \\
\hline Otras complicaciones menores (\%) & $10(8,7)$ & $0(0,0)$ & $0(0,0)$ & $3(9,4)$ & $1(16,7)$ & $4(14,8)$ & $2(6,9)$ \\
\hline
\end{tabular}

*Mediana y rango interquartil. †DS: desviación estándar. ${ }^{\S} \mathrm{NIHSS}$ : National Institutes of Health Stroke Scale.

ficas como de especialistas. Se trató exitosamente a pacientes localizados geográficamente a una distancia de hasta 823 kilómetros respecto de la UTA y están dadas las condiciones para realizar también TIV en la Isla Rapa Nui (centro activado recién en diciembre de 2018) a $3.760 \mathrm{~km}$ del área sur de Santiago.

La atención del ACVi agudo por telemedicina permite proveer la asistencia experta en lugares donde no existen neurólogos especialistas o donde el número de neurólogos no es suficiente como para una cobertura 24/7. En países y regiones donde se han desarrollado programas de telestroke se ha ampliado en forma sustancial la cobertura geográfica de acceso a la TIV con una efectividad y tasas de complicaciones similares al tratamiento con el neurólogo presencial, aportando equidad en la atención de salud ${ }^{7,8}$.

Es de vital importancia destacar que la implementación de un sistema de telemedicina implica necesariamente conocer las realidades y liderazgos de los hospitales y unidades de urgencia que serán puestos en comunicación con la UTA, de lo contrario puede ocasionar el fracaso en la implementación del sistema ${ }^{18,19}$. Una experiencia chilena previa, realizada entre la Clínica Alemana de Santiago ( $33^{\circ} 23^{\prime} 31.9956^{\prime \prime}$ S 70 34' 23.1672" $\mathrm{O})^{24}$ y el Hospital Padre Hurtado ( $33^{\circ} 33^{\prime} 7.7436^{\prime \prime}$ S 70 $\left.38^{\prime} 6.7056^{\prime \prime} \mathrm{O}\right)^{25}$ en el año 2011, no tuvo éxito y fue suspendida. En ese caso, a pesar del apoyo institucional y de una adecuada implementación tecnológica, la sobrecarga asistencial y la ausencia de un liderazgo médico en el servicio de urgencia del hospital ejecutor, se tradujeron en un mínimo uso del recurso y ninguna TIV durante el período de implementación (Dr. Arnold Hoppe, comunicación personal).

En cuanto a los resultados que se presentan, la tasa de trombolisis en el año 2018 fue de 19,2\% respecto de todas las consultas de la UTA. Se debe considerar que una tasa superior al $12 \%$ ha sido estimado en modelos de simulación como 
el indicador que tiene impacto en la reducción poblacional de la discapacidad por $\mathrm{ACVi}^{26}$.

Nuestros tiempos puertas-aguja están dentro de lo recomendado internacionalmente, con un valor promedio inferior (dentro del rango) a lo descrito en el proyecto TEMPiS, en Baviera, donde fue de $76(+-24)$ minutos ${ }^{7}$. La tasa de hemorragia cerebral sintomática en nuestros pacientes fue similar a las publicadas en series clínicas como el SITS-MOST $^{27}$ o estudios como NINDS, ECASS 2 o IST-3, que se sitúan en un rango entre 1,7\% a $7 \% \%^{3,4,28}$.

La Unidad de TeleACV, además, permitió a 8 pacientes ser derivados a un centro de mayor complejidad para trombectomía mecánica, el tratamiento de elección para el manejo de pacientes con oclusión de vasos intracerebrales de gran tamaño y quienes tienen menor respuesta a TIV, comparativamente ${ }^{29,30}$.

Nuestro estudio posee fortalezas al tratarse de un registro prospectivo de pacientes en un ambiente clínico habitual y con un número significativo de enfermos consultantes. Entre las limitaciones cabe señalar el hecho de que no existe información de los pacientes en los que no se activó el código ACV y no se cuenta con un seguimiento posterior a las primeras $24 \mathrm{~h}$, por lo que se desconoce el resultado clínico final a los 3 meses. Por la misma razón se desconoce la proporción de pacientes trombolizados cuyo estudio o evolución posterior descartó una causa vascular de los síntomas (cuadros imitadores de ACVi), situación que ha sido documentada con cierta frecuencia en servicios de urgencia ${ }^{31}$.

En conclusión, el sistema de telestroke de la Unidad de TeleACV presenta una alta tasa de TIV y tiempos puerta-aguja óptimos. Lo anterior ha contribuido a disminuir las brechas geográficas y de especialistas en nuestro país, mejorando el acceso y la equidad en la atención de salud. Para este efecto es fundamental disponer no solamente de los recursos tecnológicos y de especialistas, sino tomar ampliamente en consideración las condiciones físicas, profesionales y los liderazgos de cada uno de los respectivos hospitales ejecutores para una implementación exitosa de esta modalidad de tratamiento.

Agradecimientos: La implementación y puesta en marcha del proyecto de telemedicina de la Unidad de TeleACV del Servicio de Salud
Metropolitano Sur es resultado del apoyo de las autoridades del Servicio de Salud Metropolitano Sur, del Ministerio de Salud y del trabajo colaborativo entre el personal de todas las unidades de urgencia y unidades asociadas de cada uno de los hospitales ejecutores.

\section{Referencias}

1. Diez primeras causas de muerte en Chile entre el 2000 y 2011. Departamento de Estadísticas e Información de Salud. Ministerio de Salud. Available from: http://www. deis.cl/defunciones-y-mortalidad-por-causas/

2. Lozano R, Naghavi M, Foreman K, Lim S, Shibuya K, Aboyans V, et al. Global and regional mortality from 235 causes of death for 20 age groups in 1990 and 2010: a systematic analysis for the Global Burden of Disease Study 2010. The Lancet 2012; 380 (9859): 2095-128.

3. National Institute of Neurological Disorders and Stroke rt-PA Stroke Study Group. Tissue Plasminogen Activator for Acute Ischemic Stroke. N Engl J Med 1995; 333 (24): 1581-8.

4. Hacke W, Kaste M, Bluhmki E, Brozman M, Dávalos A, Guidetti D, et al. Thrombolysis with Alteplase 3 to 4.5 Hours after Acute Ischemic Stroke. N Engl J Med 2008; 359 (13): 1317-29.

5. Saver JL, Gornbein J, Grotta J, Liebeskind D, Lutsep H, Schwamm L, et al. Number Needed to Treat to Benefit and to Harm for Intravenous Tissue Plasminogen Activator Therapy in the 3- to 4.5-Hour Window: Joint Outcome Table Analysis of the ECASS 3 Trial. Stroke. 2009; 40 (7): 2433-7.

6. Levine SR, Gorman M. "Telestroke" : the application of telemedicine for stroke. Stroke 1999; 30 (2): 464-9.

7. Audebert HJ, Kukla C, Clarmann von Claranau S, Kühn J, Vatankhah B, Schenkel J, et al. Telemedicine for Safe and Extended Use of Thrombolysis in Stroke: The Telemedic Pilot Project for Integrative Stroke Care (TEMPiS) in Bavaria. Stroke 2005; 36 (2): 287-91.

8. Ribo M, Molina CA, Pedragosa A, Sanclemente C, Santamarina E, Rubiera M, et al. Geographic Differences in Acute Stroke Care in Catalunya: Impact of a Regional Interhospital Network. Cerebrovasc Dis 2008; 26 (3): 284-8

9. Audebert HJ, Schultes K, Tietz V, Heuschmann PU, Bogdahn U, Haberl RL, et al. Long-Term Effects of Specialized Stroke Care With Telemedicine Support in Community Hospitals on Behalf of the Telemedical Project for Integrative Stroke Care (TEMPiS). Stroke 2009; 40 (3): 902-8.

10. Silva GS, Farrell S, Shandra E, Viswanathan A, 
Schwamm LH. The Status of Telestroke in the United States: A Survey of Currently Active Stroke Telemedicine Programs. Stroke 2012; 43 (8): 2078-85.

11. Nardetto L, Dario C, Tonello S, Brunelli MC, Lisiero M, Carraro MG, et al. A one-to-one telestroke network: the first Italian study of a web-based telemedicine system for thrombolysis delivery and patient monitoring. Neurol Sci 2016; 37 (5): 725-30.

12. Legris N, Hervieu-Bègue $M$, Daubail B, Daumas A, Delpont B, Osseby G-V, et al. Telemedicine for the acute management of stroke in Burgundy, France: an evaluation of effectiveness and safety. Eur J Neurol 2016; 23 (9): 1433-40.

13. Chalouhi N, Dressler JA, Kunkel ESI, Dalyai R, Jabbour P, González LF, et al. Intravenous Tissue Plasminogen Activator Administration in Community Hospitals Facilitated by Telestroke Service: Neurosurgery 2013; 73 (4): 667-72.

14. Schwamm LH, Holloway RG, Amarenco P, Audebert HJ, Bakas T, Chumbler NR, et al. A Review of the Evidence for the Use of Telemedicine Within Stroke Systems of Care: A Scientific Statement From the American Heart Association/American Stroke Association. Stroke 2009; 40 (7): 2616-34.

15. Peñaloza-Ramos MC, Sheppard JP, Jowett $S$, Barton $P$, Mant J, Quinn T, et al. Cost-Effectiveness of Optimizing Acute Stroke Care Services for Thrombolysis. Stroke 2014; 45 (2): 553-62.

16. Wechsler LR, Demaerschalk BM, Schwamm LH, Adeoye OM, Audebert HJ, Fanale CV, et al. Telemedicine Quality and Outcomes in Stroke: A Scientific Statement for Healthcare Professionals From the American Heart Association/American Stroke Association. Stroke [Internet]. 2017; 48 (1). Available from: https://www. ahajournals.org/doi/10.1161/STR.0000000000000114 [citado el 4 de marzo de 2019].

17. Powers WJ, Rabinstein AA, Ackerson T, Adeoye OM, Bambakidis NC, Becker K, et al. 2018 Guidelines for the Early Management of Patients With Acute Ischemic Stroke: A Guideline for Healthcare Professionals From the American Heart Association/American Stroke Association. Stroke [Internet]. 2018; 49 (3). Available from: https://www.ahajournals.org/doi/10.1161/ STR.0000000000000158 [citado el 4 de marzo de 2019].

18. Gulube SM, Wynchank S. Telemedicine in South Africa: Success or failure? J Telemed Telecare 2001; 7 (2 suppl): 47-9.

19. Ranta A, Busch S. Impact of discontinuation of telestroke: the Nelson experience. N Z Med J 2018; 131 (1477): 29-34.

20. Kothari RU, Pancioli A, Liu T, Brott T, Broderick J.
Cincinnati Prehospital Stroke Scale: reproducibility and validity. Ann Emerg Med 1999; 33 (4): 373-8.

21. Lyden PD, Lu M, Levine SR, Brott TG, Broderick J, NINDS rtPA Stroke Study Group. A modified National Institutes of Health Stroke Scale for use in stroke clinical trials: preliminary reliability and validity. Stroke 2001; 32 (6): 1310-7.

22. Pexman JH, Barber PA, Hill MD, Sevick RJ, Demchuk AM, Hudon ME, et al. Use of the Alberta Stroke Program Early CT Score (ASPECTS) for assessing CT scans in patients with acute stroke. AJNR Am J Neuroradiol 2001; 22 (8): 1534-42.

23. An International Randomized Trial Comparing Four Thrombolytic Strategies for Acute Myocardial Infarction. N Engl J Med 1993; 329 (10): 673-82.

24. Google Maps. Ubicación de Clínica Alemana de Santiago [Internet]. Available from: https://goo.gl/maps/ ZbupXbHTxz32 [citado el 4 de marzo de 2019].

25. Google Maps. Ubicación del Hospital Padre Hurtado de Santiago [Internet]. Available from: https://goo.gl/maps/ nRbHxNviGqS2 [citado el 4 de marzo de 2019].

26. Hoffmeister L, Lavados PM, Mar J, Comas M, Arrospide A, Castells X. Minimum intravenous thrombolysis utilization rates in acute ischemic stroke to achieve population effects on disability: A discrete-event simulation model. J Neurol Sci 2016; 365: 59-64.

27. Wahlgren N, Ahmed N, Dávalos A, Ford GA, Grond M, Hacke W, et al. Thrombolysis with alteplase for acute ischaemic stroke in the Safe Implementation of Thrombolysis in Stroke-Monitoring Study (SITS-MOST): an observational study. The Lancet 2007; 369 (9558): 27582.

28. The benefits and harms of intravenous thrombolysis with recombinant tissue plasminogen activator within $6 \mathrm{~h}$ of acute ischaemic stroke (the third international stroke trial [IST-3]): a randomised controlled trial. The Lancet 2012; 379 (9834): 2352-63.

29. Saver JL, Goyal M, van der Lugt A, Menon BK, Majoie CBLM, Dippel DW, et al. Time to Treatment With Endovascular Thrombectomy and Outcomes From Ischemic Stroke: A Meta-analysis. JAMA 2016; 316 (12): 1279.

30. Goyal M, Menon BK, van Zwam WH, Dippel DWJ, Mitchell PJ, Demchuk AM, et al. Endovascular thrombectomy after large-vessel ischaemic stroke: a meta-analysis of individual patient data from five randomised trials. The Lancet 2016; 387 (10029): 1723-31.

31. Brunser AM, Illanes S, Lavados PM, Muñoz P, Cárcamo D, Hoppe A, et al. Exclusion Criteria for Intravenous Thrombolysis in Stroke Mimics: An Observational Study. J Stroke Cerebrovasc Dis 2013; 22 (7): 1140-5. 